\title{
Jejunal hemorrhage due to hemolymphangioma successfully detected and treated by double-balloon enteroscopy
}

\author{
Yuki Ito ${ }^{1}$, Tatsuya Osuga ${ }^{1}$, Kentaro Kawasaki ${ }^{1}$, and Y Ikura ${ }^{1}$ \\ ${ }^{1}$ Aijinkai Takatsuki General Hospital
}

October 25, 2021

\begin{abstract}
Lymphangiomas are a common benign neoplasm, which can develop in the digestive tracts. Hemorrhagic jejunal tumors are relatively rare and diagnostic challenge. We report herein a case of hemorrhagic jejunal hemolymphangioma successfully diagnosed and treated by double-balloon enteroscopy.

CLINICAL IMAGE

Jejunal hemorrhage due to hemolymphangioma successfully detected and treated by doubleballoon enteroscopy

Yuki Ito $^{1}$ | Tatsuya Osuga ${ }^{1}$ Kentaro Kawasaki ${ }^{2}$ | Yoshihiro Ikura ${ }^{3}$

Departments of ${ }^{1}$ Gastroenterology, ${ }^{2}$ Surgery and ${ }^{3}$ Pathology, Takatsuki General Hospital,

1-3-13, Kosobecho, Takatsuki 569-1192, Japan

Correspondence

Yoshihiro Ikura, MD, Department of Pathology, Takatsuki General Hospital, 1-3-13, Kosobecho, Takatsuki 569-1192, Japan. E-mail:ikura@ajk.takatsuki-hp.or.jp

Fax: +81726823834 Tel: +81726813801

Running title

Enteroscopic hemostasis of lymphangioma

\section{Abstract}

Lymphangiomas are a common benign neoplasm, which can develop in the digestive tracts. Hemorrhagic jejunal tumors are relatively rare and diagnostic challenge. We report herein a case of hemorrhagic jejunal hemolymphangioma successfully diagnosed and treated by double-balloon enteroscopy.
\end{abstract}

\section{KEYWORDS}

Intestinal hemorrhage; Hemolymphangioma; Enteroscopic hemostasis

\section{Key Clinical Message}

A small jejunal lymphangioma is diagnostic challenge and can evoke life-threatening hemorrhage. Doubleballoon enteroscopy is an useful tool for its diagnosis and treatment.

\section{1 | CASE PRESENTATION}


A 74-year-old woman receiving an anticoagulant for atrial fibrillation was admitted to our hospital because of persistent abdominal pain and melena. Laboratory test results indicated severe anemia (hemoglobin, 3.0 $\mathrm{g} / \mathrm{dL}$ ). Upper gastrointestinal endoscopy and colonoscopy were performed, but no hemorrhagic lesion was identified. However, as tarry enteric fluid flowing out from ileocecal valve was observed in the colonoscopic examination, we speculated that the bleeding point existed at small intestin. Double-balloon enteroscopy was performed, and as was expected, a small $(\varnothing 6 \mathrm{~mm})$ polypoid lesion with active bleeding was found at the jejunum (Fig. 1a). Endoscopic clipping was immediately done for hemostasis (Fig. 1b). Subsequently, partial jejunectomy was conducted to prevent re-bleeding. The surgical specimen had a tiny hemorrhagic lesion (Fig. 2a), which histologically consisted of aggregation of dilated lympho-vascular channels in the subepithelial region (Fig. 2b). The final pathologic diagnosis was jejunal hemolymphangioma. To date, no recurrence of melena has been reported and her hematological data have been improving (hemoglobin, $8.9 \mathrm{~g} / \mathrm{dL}$ ).

\section{2 | DISCUSSION AND CONCLUSION}

Lymphangiomas are a common benign neoplasm, which can develop in the digestive tracts. Bleeding is an important manifestation in usually [?]20 mm tumors. ${ }^{1,2}$ Our case suggests that lymphangiomas, even if small, can cause life-threatening gastrointestinal hemorrhage.

\section{ACKNOWLEDGMENTS}

None.

\section{CONFLICT OF INTEREST}

None.

\section{AUTHOR CONTRIBUTIONS}

Y.Ito was responsible for writing the initial draft of the manuscript. Y.Ikura was responsible for conception, design, drafting, image modification, and finalizing the manuscript. KK was responsible for surgery and image modification. TO was responsible for design and finalizing. All authors read and approved the final manuscript.

\section{ETHICAL STATEMENT}

Informed consent for publication and related images has been obtained from the patient.

\section{DATA AVAILABILITY STATEMENT}

No datasets were generated or analyzed during this case report.

\section{ORCID}

Yoshihiro Ikura (ID) https://orcid.org/0000-0001-8599-2245

\section{REFERENCE}

1. Ikura Y, Hashimoto T, Takamine Y, et al. Lymphangioma of the duodenum: report of a case. Surg Today 1994;24:160-163.

2. Xiao NJ, Ning SB, Li T, et al. Small intestinal hemolymphangioma treated with enteroscopic injection sclerotherapy: A case report and review of literature. World J Gastroenterol2020;26:1540-1545.

\section{Figure legends}

FIGURE 1 Representative images of double-balloon enteroscopy. (a ) A small polypoid lesion with active bleeding is seen (arrowheads). (b ) Endoscopic clipping successfully stopped bleeding.

FIGURE 2 Pathologic findings of the surgical specimen. (a ) A small hemorrhagic polypoid lesion was confirmed in macroscopic observation (arrowheads). In a cut surface, the hemorrhagic change is located only at the apex of the lesion (inset). (b ) A microscopic image of the apex portion shows aggregation of dilated 
vessels filled with lymphatic fluid and blood accompanied by extravasation of erythrocytes. (Hematoxylineosin stain, x40 magnification)
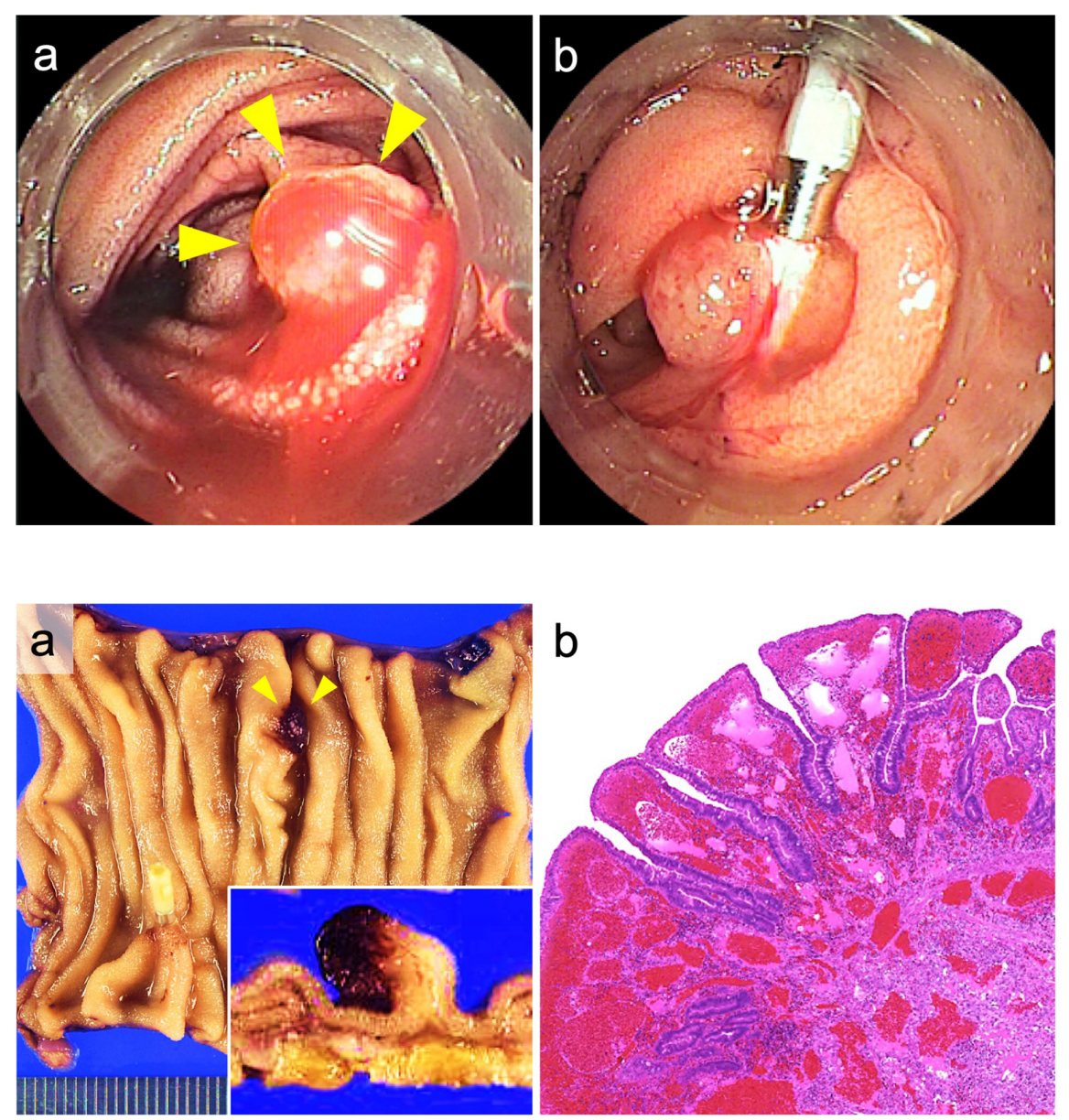